2. Douglas JD. Patterns of change following parent death in midlife adults. Omega 1991;22:123-37.

3. Donoho CJ, Bonanno GA, Porter B, Kearney L, Powell TM. A decade of war: prospective trajectories of posttraumatic stress disorder symptoms among deployed US military personnel and the influence of combat exposure. Am J Epidemiol 2017;186: 1310-8.

DOI: 10.1056/NEJMc1809967

THE AUTHORS REPLY: Yusufov and colleagues suggest that we should have tested a brief, complex, psychologically focused intervention to mitigate surrogates' psychological distress. Although we agree that this type of intervention should be developed and tested in the ICU setting to reduce surrogates' psychological distress, we chose a broader focus for the PARTNER intervention because our intention was to improve two different domains of outcomes: surrogates' long-term psychological outcomes and the quality of decisions made for incapacitated, critically ill patients. On the basis of the available evidence when we designed the trial, ${ }^{1}$ we hypothesized that we could improve both outcome domains with one intervention focused on improving communication and decision support. Although the PARTNER intervention improved measures of decision quality and decreased the duration of intensive treatment among dying patients, our results suggest that a different kind of intervention may be needed to improve surrogates' long-term psychological outcomes.

Akca characterizes the PARTNER intervention as largely composed of scheduled interdisciplinary family meetings. We respectfully disagree with this description of the intervention, because it fails to recognize that this was a complex behavioral intervention that included extensive daily encounters between the PARTNER nurses and surrogates, not just interdisciplinary family meetings. Akca raises the question of whether the results of the primary outcome (surrogates' HADS score at 6 months) were biased in favor of the intervention because of the longer duration in the intervention group than in the control group between a patient's death and the 6-month outcome assessment. To assess this, we restricted the published prespecified multivariable analysis to surrogates of patients who died and added a covariate that quantified the number of days between the patient's death and the 6-month assessment of the HADS score; this analysis also showed no significant difference between the control group and the intervention group in surrogates' HADS scores at 6 months (adjusted mean score, 11.9 and 12.3 , respectively; $\mathrm{P}=0.58$ ).

Mao and colleagues suggest that we should have measured more baseline psychological risk factors and that we should have assessed surrogates' psychological distress at multiple time points. We agree that these data would have allowed important insights into the contributors to and trajectory of surrogates' psychological symptoms. However, we opted for a parsimonious measurement strategy because of the pragmatic nature of the trial and the need to minimize respondent burden in this trial population, since these respondents were frequently bereaved or facing substantial caregiving burdens.

Douglas B. White, M.D.

Praewpannarai Buddadhumaruk, R.N., M.S. Robert M. Arnold, M.D.

University of Pittsburgh School of Medicine

Pittsburgh, PA

whitedb@upmc.edu

Since publication of their article, the authors report no further potential conflict of interest.

1. Wendler D, Rid A. Systematic review: the effect on surrogates of making treatment decisions for others. Ann Intern Med 2011;154:336-46.

DOI: 10.1056/NEJMc1809967

\title{
Ambulatory Blood Pressure and Mortality
}

TO THE EDITOR: Banegas et al. (April 19 issue) ${ }^{1}$ claim to have found that ambulatory blood pressure is a better predictor of mortality than blood pressure measured in the clinic (clinic blood pressure). However, this study does not really address the critical question of how much better.
My colleagues and I conducted a study that was based on population surveys and that used clinic and ambulatory blood-pressure data from 7927 persons. $^{2}$ The results of our analyses were similar to those of the study conducted by Banegas et al.; we found a significant change in 
the area under the receiver-operating-characteristic curve in several subgroups. However, the actual difference in predictive value between clinic blood pressure alone and clinic blood pressure plus ambulatory blood pressure was negligible, and we concluded that clinic blood pressure was a sufficient tool for population screening. It is important to determine the actual magnitude of the improvement in predictive value before recommending the widespread use of ambulatory blood-pressure monitoring - a determination that cannot be made with the use of the $\mathrm{C}$ statistic or with hazard ratios.

Christian Torp-Pedersen, M.D., D.M.S.C.
Aalborg University
Aalborg, Denmark
ctp@heart.dk
No potential conflict of interest relevant to this letter was re-
ported.

1. Banegas JR, Ruilope LM, de la Sierra A, et al. Relationship between clinic and ambulatory blood-pressure measurements and mortality. N Engl J Med 2018;378:1509-20.

2. Mortensen RN, Gerds TA, Jeppesen JL, Torp-Pedersen C. Office blood pressure or ambulatory blood pressure for the prediction of cardiovascular events. Eur Heart J 2017;38:3296-304.

DOI: $10.1056 / N E J M c 1809851$

TO THE EDITOR: Banegas et al. confirm the importance of 24-hour ambulatory blood-pressure monitoring. However, some of the findings were unexpected, such as the finding that cardiovascular risk was similar among untreated normotensive persons and patients with treated normalized hypertension, as well as the tendency toward a risk among persons with treated whitecoat hypertension that was lower than the risk among those with untreated white-coat hypertension (Table 3, and Figure S4 in the Supplementary Appendix, available with the full text of the article at NEJM.org) - results that are incompatible with previous publications. ${ }^{1,2}$

Methodologic issues may explain these results. First, Banegas et al. pooled treated and untreated persons together in one model and adjusted for the number of antihypertensive drugs, thereby masking the higher risk among treated persons than among untreated persons who had similar blood pressures. Second, Banegas et al. did not account for the clustering of end points within centers, which can lead to unexpected results. ${ }^{3}$ In addition, the study population consisted of high-risk patients who had been referred for ambulatory blood-pressure monitoring, the quality of the clinic blood-pressure readings remains to be established, and nonfatal end points were not considered - three issues that limit the generalizability of the study.

Lutgarde Thijs, M.Sc.

Wen-Yi Yang, M.D.

Jan A. Staessen, M.D., Ph.D.

University of Leuven

Leuven, Belgium

lutgarde.thijs@kuleuven.be

No potential conflict of interest relevant to this letter was reported.

1. Franklin SS, Thijs L, Hansen TW, et al. Significance of white-coat hypertension in older persons with isolated systolic hypertension: a meta-analysis using the International Database on Ambulatory Blood Pressure Monitoring in Relation to Cardiovascular Outcomes population. Hypertension 2012;59:564-71.

2. Lawlor DA, Kim L, Morris R, Amuzu A, Whincup P, Ebrahim S. Survival with treated and well-controlled blood pressure: findings from a prospective cohort study. PLoS One 2011;6(4):e17792. 3. Abo-Zaid G, Guo B, Deeks JJ, et al. Individual participant data meta-analyses should not ignore clustering. J Clin Epidemiol 2013;66:865-73.

DOI: 10.1056/NEJMc1809851

TO THE EDITOR: Banegas et al. found that 24-hour systolic ambulatory blood pressure was more strongly associated with all-cause mortality and cardiovascular mortality than clinic systolic blood pressure. Although this study is informative, it lacks data regarding one of the most important findings regarding the monitoring of ambulatory blood pressure - the pattern of dipping status (blood pressure that dips [i.e., is lower during the night than during the day], that does not dip, that dips extremely, or that rises). ${ }^{1}$ Dipping status is associated with cardiovascular outcomes in a variety of ways. ${ }^{2}$

First, patients whose blood pressure does not dip are potentially exposed to higher blood-pressure loads than patients whose blood pressure dips. So, if the question is whether the pattern of the diurnal rhythm of blood pressure matters, comparisons should be made between patients whose blood pressure dips and those with the same 24-hour blood-pressure level whose blood pressure does not dip.

Second, an exaggerated blood-pressure surge in the morning is associated with worse cardiovascular outcomes. ${ }^{3}$ Third, extreme dipping in blood pressure can be associated with ischemic damage and an increased risk of cardiovascular events. ${ }^{4}$ Thus, information regarding these patterns - which can be detected by ambulatory 
blood-pressure monitoring - would be of interest and may further support the role of such monitoring in predicting cardiovascular outcomes.

Baris Afsar, M.D.

Suleyman Demirel University

Isparta, Turkey

afsarbrs@yahoo.com

No potential conflict of interest relevant to this letter was reported.

1. Pickering TG. Should we be evaluating blood pressure dipping status in clinical practice? J Clin Hypertens (Greenwich) 2005;7:178-82.

2. Sarigianni M, Dimitrakopoulos K, Tsapas A. Non-dipping status in arterial hypertension: an overview. Curr Vasc Pharmacol 2014;12:527-36.

3. Turak O, Afsar B, Ozcan F, et al. Relationship between elevated morning blood pressure surge, uric acid, and cardiovascular outcomes in hypertensive patients. J Clin Hypertens (Greenwich) 2014;16:530-5.

4. Kario K, Shimada K. Risers and extreme-dippers of nocturnal blood pressure in hypertension: antihypertensive strategy for nocturnal blood pressure. Clin Exp Hypertens 2004;26:177-89.

DOI: $10.1056 /$ NEJMc1809851

THE AUTHORS REPLY: Torp-Pedersen makes a reasonable point about assessing the magnitude of the improvement in mortality prediction by using ambulatory blood pressure in addition to clinic blood pressure. We have now compared, for each study participant, the absolute risk of death from cardiovascular causes with estimates from a model that included cardiovascular risk factors, the 24hour ambulatory systolic pressure, and the clinic systolic pressure with the risk that was estimated with the same model without ambulatory blood pressure. The median difference in the 4.7-year absolute risk among all the patients was 0.1 percentage points (interquartile range, -0.3 to 0.9 ), but $14 \%$ of the study participants had a difference of more than 2 percentage points in the risk of death from cardiovascular causes. Given that among these $14 \%$ of participants the absolute cardiovascular mortality was $2.3 \%$ (204 deaths from cardiovascular causes $\div 8786$ participants), the improvement in mortality prediction among individual patients with the use of ambulatory blood pressure was substantial. We advocate clinic blood pressure as a screening tool, but ambulatory blood pressure is preferred for confirming the diagnosis and for assessing the quality of blood-pressure control. This is now supported by the new European hypertension guidelines. ${ }^{1}$

In response to Thijs et al.: we have now performed the main analyses without adjustment for the number of antihypertensive drugs. For exam- ple, in model 2 in our study, the hazard ratio among patients with controlled hypertension, as compared with those with normotension, was 0.99 (95\% confidence interval [CI], 0.76 to 1.30 ) for death from any cause and 1.34 (95\% CI, 0.84 to 2.14) for death from cardiovascular causes. The hazard ratios among patients with white-coat hypertension and those with white-coat uncontrolled hypertension, as compared with those with normotension, were 1.77 (95\% CI, 1.37 to 2.30) and 1.30 (95\% CI, 1.02 to 1.66), respectively, for death from any cause and 1.94 (95\% CI, 1.21 to 3.11) and 1.46 (95\% CI, 0.93 to 2.30), respectively, for death from cardiovascular causes. Thus, the risk of death among participants with controlled hypertension did not differ significantly from the risk among participants with normotension, and the tendency toward a lower risk among patients with treated white-coat hypertension than those with untreated white-coat hypertension was still present. Our results showing that white-coat hypertension is not benign are consistent with some, ${ }^{2,3}$ but not all, ${ }^{4}$ previous studies.

We do not think that these results are due to ignoring the clustering of patients in the analyses, because the number of clusters was large ( $>200$ primary care centers participated) and the analyses were adjusted for a large number of covariates. In the article, we suggested that the tendency toward lower mortality was probably because participants with treated and controlled hypertension were more likely to have frequent follow-up visits, medication adjustments, and treatment of concomitant conditions that may contribute to the risk of death than patients with untreated hypertension.

As requested by Afsar, we report the dipping status $^{5}$ of the study participants. A total of $41.1 \%$ of the participants had blood pressure that dipped (night:day blood-pressure ratio, 0.8 to 0.9 ), $39.5 \%$ had blood pressure that dipped in a reduced fashion (ratio, 0.9 to 1 ), $6.7 \%$ had blood pressure that dipped extremely (ratio, <0.8), and $12.7 \%$ had blood pressure that did not dip or that rose (ratio, $\geq 1$ ). Hazard ratios for death from cardiovascular causes according to systolic dipping status (with dipping as the reference), with adjustment for demographic characteristics, cardiovascular risk factors, and 24-hour and clinic systolic pressures, were as follows: 1.17 (95\% CI, 1.02 to 1.34 ) among patients with reduced dipping, 1.12 (95\% CI, 0.87 to 1.45) among those 
with extreme dipping, and 1.34 (95\% CI, 1.14 to 1.57) among those with no dipping or with rising blood pressure.

José R. Banegas, M.D.

Universidad Autónoma de Madrid

Madrid, Spain

joseramon.banegas@uam.es

Luis M. Ruilope, M.D.

Hospital Universitario 12 de Octubre

Madrid, Spain

Bryan Williams, M.D.

University College London

London, United Kingdom

Since publication of their article, the authors report no further potential conflict of interest.
1. Williams B, Mancia G, Spiering W, et al. $2018 \mathrm{ESC} / \mathrm{ESH}$ guidelines for the management of arterial hypertension. Eur Heart J 2018;39:3021-104.

2. Huang $\mathrm{Y}$, Huang $\mathrm{W}$, Mai $\mathrm{W}$, et al. White-coat hypertension is a risk factor for cardiovascular diseases and total mortality. J Hypertens 2017;35:677-88.

3. Cuspidi C, Tadic M, Mancia G, Grassi G. White-coat hypertension: the neglected subgroup in hypertension. Korean Circ J 2018;48:552-64.

4. Franklin SS, Thijs L, Hansen TW, et al. Significance of white-coat hypertension in older persons with isolated systolic hypertension: a meta-analysis using the International Database on Ambulatory Blood Pressure Monitoring in Relation to Cardiovascular Outcomes population. Hypertension 2012;59:564-71.

5. Parati G, Stergiou G, O'Brien E, et al. European Society of Hypertension practice guidelines for ambulatory blood pressure monitoring. J Hypertens 2014;32:1359-66.

DOI: 10.1056/NEJMc180985]

\section{Intussusception after Rotavirus Vaccination in Africa}

TO THE EDITOR: Tate et al. (April 19 issue) ${ }^{1}$ reported the incidence of intussusception after monovalent rotavirus vaccination among 717 infants from seven African countries. They concluded that the risk of intussusception after rotavirus vaccination was not higher than the background risk of intussusception in the African countries they examined. I wonder why countries such as South Africa, Morocco, and Sudan were not included, given that the earliest introductions of rotavirus vaccination were in these countries (in 2009, 2010, and 2011, respectively). ${ }^{2}$ I am also concerned that a sample size of 717 infants may not be statistically sufficient for an analysis of an uncommon condition like intussusception. The authors did not mention some important causes of and risk factors for intussusception, such as infections, congenital anomalies, and Henoch-Schönlein purpura. Furthermore, mortality from intussusception is still high in Africa. Many infants with intussusception in Africa die before reaching a hospital ${ }^{2}$; in addition, skill in the radiologic diagnosis of the condition is often lacking, especially in non-tertiary care hospitals. For these reasons, I think that the rate of naturally occurring as well as rotavirusrelated intussusception among infants may still be underreported in Africa.

Erwa Elmakki, M.D.

Jazan University

Jazan, Saudi Arabia
No potential conflict of interest relevant to this letter was reported.

1. Tate JE, Mwenda JM, Armah G, et al. Evaluation of intussusception after monovalent rotavirus vaccination in Africa. $\mathrm{N}$ Engl J Med 2018;378:1521-8.

2. Sow SO, Steele AD, Mwenda JM, Armah GE, Neuzil KM. Reaching every child with rotavirus vaccine: report from the 10th African rotavirus symposium held in Bamako, Mali. Vaccine 2017; 35:5511-8

DOI: $10.1056 /$ NEJMc1 807645

THE AUTHORS REPLY: Because our evaluation focused on sub-Saharan African countries, the northern African countries of Morocco and Sudan were not included. Since the risk of intussusception after rotavirus vaccination in lower-income countries could be different, we did not include middle-income South Africa. We analyzed 717 cases, and our study had more than $80 \%$ power to detect a relative incidence of intussusception of 2.5 or more within 1 to 7 days after the first dose. This is the largest postlicensure evaluation of intussusception to date. Since our goal was to assess the possible association between rotavirus vaccination and intussusception, we did not investigate other risk factors for or causes of intussusception. The self-controlled case-series method automatically controls for confounding by fixed risk factors. We may not have captured all cases of intussusception and cannot estimate populationbased rates of the condition. However, because the self-controlled case-series method depends on 